\title{
SPATIAL DEPENDENCE OF RANDOM SETS AND ITS APPLICATION TO DISPERSION OF BARK BEETLE INFESTATION IN A NATURAL FOREST
}

\author{
MARKUS KAUTZ ${ }^{1}$, JOCHEN DÜLL ${ }^{2}$ AND JOACHIM OHSER ${ }^{2}$ \\ ${ }^{1}$ Technische Universität München, Lehrstuhl für Tierökologie, Hans-Carl-von-Carlowitz-Platz 2, D-85354 \\ Freising; ${ }^{2}$ Hochschule Darmstadt, Fachbereich Mathematik und Naturwissenschaften, Holzhofallee 38, \\ D-64295 Darmstadt \\ e-mail: kautz@wzw.tum.de,ohser@fh-darmstadt.de \\ (Accepted August 8, 2011)
}

\begin{abstract}
A large spatio-temporal data set monitoring the annual progress of bark beetle infestation in the Bavarian Forest National Park (Germany) is statistically analysed by means of complex image analysis algorithms. The infestation data were obtained by color-infrared (CIR) aerial image interpretation and cover 10 subsequent years (2001-2010). Newly emerged infestation patches are hypothesized as spatially correlated to locations of previous year's infestation. Both areas, source patches and subsequently emerged patches, are considered as two disjoint random sets. Their spatio-temporal dependence is analysed by two methods: the classical approach based on the measurement of cross-covariance functions, and a second one based on nearest neighbor distances. The resulting characteristics can be interpreted as pre-disposition probabilities of bark beetle infestation depending on distance to sources. Both methods show a strong short-range preference, which decreases with increasing distances.
\end{abstract}

Keywords: cross-covariance function, ecological data, Ips typographus, pre-disposition, image analysis, Euclidean distance transform, Fast Fourier transform.

\section{INTRODUCTION}

The European spruce bark beetle Ips typographus L. (Coleoptera, Curculionidae, Scolytinae) is one of the most severe pest species killing living spruce trees (Picea abies L. Karst., Pinaceae) in Europe. Its horizontal larval galleries and maturation feeding in adult stage girdle tree's phloem and destroy the cambium. The trees which are infested by bark beetles are going to die. As a consequence, the next emerging beetle generation is forced to disperse from their breeding tree in order to find new suitable host trees (Lieutier et al., 2004). The complex process of beetle dispersal and their habitat selection still offers several unsolved questions, which are the focus of recent research (Wermelinger, 2004). Generally, dispersal of bark beetles within a habitat is assumed to be influenced by various host and site specific factors, such as tree vigor, species composition or solar radiation (Jakus, 1995; Netherer and Nopp-Mayr, 2005). Since these factors do not show spatial homogeneity at landscape scale, the distribution of infestation patches reflects the most preferred localities. Nevertheless, dispersal distances are generally determined by a combination of the specific flight capacity and host selection behavior of the individuals, which vary within populations (Wallin and Raffa, 2004;
Williams and Robertson, 2008; Hawkes, 2009), as well as of the spatial availability of suitable habitats. When attacking living trees a pheromone mediated mass attack is necessary to overcome the tree's defense (Byers, 2004). Therefore, a successful attack leading to an infestation event is generally more likely in the vicinity of source patches where enough beetles are present. Otherwise, a spatial spread is crucial to maintain long-term population stability, both through avoidance of inbreeding and the escape from antagonists (Byers, 2004).

The Bavarian Forest National Park (Germany) provides a sound data base for long-term investigations of bark beetle infestation spread. Due to the protected status, forest management measures were not applied in the core zones and natural dynamics are allowed to develop undisturbed by humans. Simultaneously, annual aerial surveys provide high-resolution colorinfrared (CIR) images which document well the spatio-temporal progress of infestations over the years (Heurich et al., 2010; Kautz et al., 2011).

Both, for management purposes as well as to understand the dispersal behavior of Ips typographus the question arises, how subsequent infestation patches are spatially correlated. Researchers in the field of ecology recently approached that task by GISbased distance methods, suggesting a strong mutual 
dependence between infestation patches of subsequent years (Wichmann and Ravn, 2001; Kautz et al., 2011). Nevertheless, advanced techniques from the fields of image analysis and spatial statistics presented in this article can provide an appropriate alternative to common GIS tools applicable to ecological data such as bark beetle infestation. They are more flexible, provide a broader range of characteristics and can use full image resolution. We consider two different approaches characterizing spatial dependence of random sets: (i) the classical spatial crosscorrelation, and (ii) the distance method as nearest neighbor measurement. Several challenges arising in the computation of cross-covariance functions via frequency space are addressed in detail. Furthermore, due to the irregular shapes of the observation windows, special attention must be payed to the handling of edge effects.

Spatial cross-correlation is an extension of the well-known concept of linear correlation for univariate random variables. It has been previously studied for marked point fields (Stoyan, 1984a;b) and between a random point field, a random fiber system and random sets, where the points are sites of trees, the fibers are river courses, and the random sets are regions of specific soil types (Stoyan and Ohser, 1982). Between such structures there may exist various relationships, e.g., attraction between the point sites and the river courses or inhibition between sites and soil types. For further investigation of cross-correlation applied to data from ecology and geoscience see Agterberg and Fabbri (1979), Reich et al. (1994) and Duffy and Hughes-Clarke (2005).

Single infestation patches strongly vary in their size and shape (Kautz et al., 2011, see also Fig. 1), and systems of infestation patches usually form very irregular random sets. Therefore, it seems to be not convenient to model a system of infestation patches by a marked point process. A better (and probably more natural) model is a random set with positive area fraction.

Spatial cross-correlation of any two random sets can be introduced by the cross-correlation measure of the random area measures associated with the random

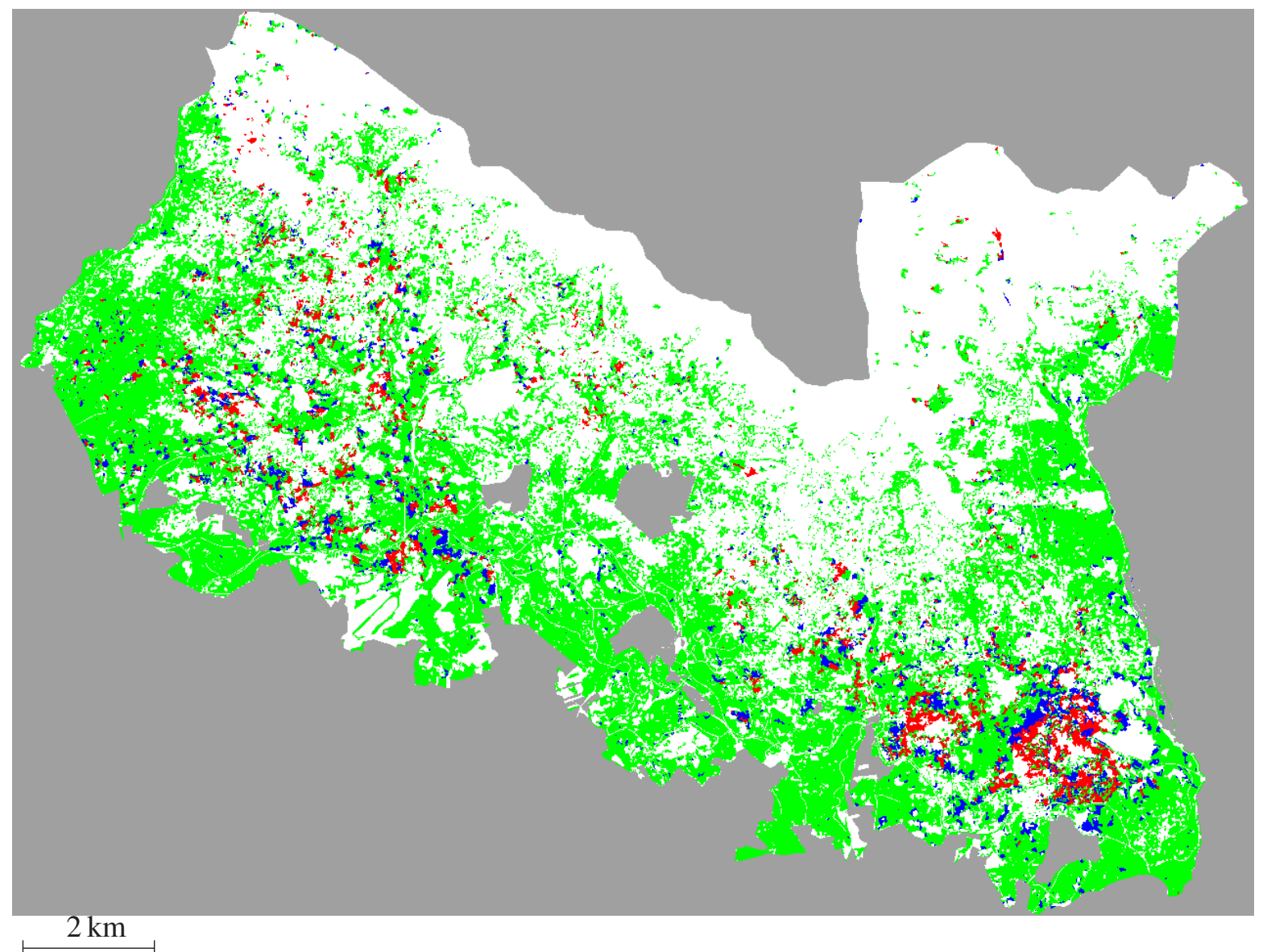

Fig. 1. Data example for one time step: shown are the infestation of 2007 (source) in red, the infestation of 2008 (target) in blue, and the susceptible forest area to potential 2008 infestation in green and blue. The frame of the study site at Bavarian Forest National Park is colored in grey. 
sets. A fundamental theoretical introduction to crosscorrelation measures is given in Stoyan and Ohser (1984). In the present article, mutual dependence of random sets is characterized by their cross-covariance function, which is the probability that two points, at a given distance from each other, are both inside the sets.

In contrast to the cross-covariance function, we also consider nearest neighbor distances between the sets. More precisely, we measure the shortest distance from a point randomly chosen in the first set to the second set. Obviously, the kind of this information differs from that contained in the crosscovariance function. The subset of the second set closest to a subset of the first one can be seen as a zone of influence. This seems to be a more natural description of the dispersal behavior of bark beetles, which spread out to find susceptible trees in their close vicinity. The use of nearest neighbor distances for investigating stochastic dependence (or independence) of random point fields was suggested by Ma et al. (2006), Baddeley and Turner (2006) and Illian et al. (2008, pp. 437 and 443). In Rack et al. (2008) a distance method is applied in order to characterize spatial dependence of constituents of materials structures.

A specific problem addressed in this paper is that the disjointness of the two random areas induces their dependence even if subsequent infestation would be independent of that in the previous year, i.e., the distribution of the infestated area inside the susceptible forest area is independent of the source.

\section{CHARACTERIZATION OF MUTUAL DEPENDENCE OF RANDOM SETS}

In the following we introduce two different methods for describing spatial dependence of specified areas within a forest. Mathematically these areas are modelled as random sets $\Phi, \Psi, \Xi$ in the twodimensional space $\mathbb{R}^{2}$. Furthermore, we assume that these random sets are almost surely locally polyconvex (i.e., the realization can be represented as finite unions of compact and convex sets) and fulfill certain regularity conditions, see Definition 9.2.1 in Schneider and Weil (2008). Finally, the random sets are assumed to be jointly macroscopically homogeneous, i.e., $\mathbb{P}(\Phi \in A, \Psi \in B, \Xi \in C)$ is invariant under diagonal shifts,

$$
\begin{aligned}
\mathbb{P}(\Phi \in A, \Psi \in B, \Xi \in C)= \\
\mathbb{P}(\Phi \in A+x, \Psi \in B+y, \Xi \in C+z)
\end{aligned}
$$

for all $x, y, z \in \mathbb{R}^{2}$ with $x=y=z$ and for all systems $A$, $B$ and $C$ of closed sets in $\mathbb{R}^{2}$.

\section{SPATIAL CROSS-CORRELATION}

Because of the joint macroscopic homogeneity of $\Phi$ and $\Psi$ the probability that the point $x$ is in $\Phi$ while $y$ is in $\Psi$ depends on only the difference $y-x$, i.e., $\mathbb{P}(x \in \Phi, y \in \Psi)$ can be seen as a function of $h=y-x$. The cross-covariance function $\operatorname{cov}_{\Phi, \Psi}$ of the random sets $\Phi$ and $\Psi$ with positive volume fractions $A_{A}(\Phi)$ and $A_{A}(\Psi)$ is defined as

$$
\begin{aligned}
\operatorname{cov}_{\Phi, \Psi}(h)=\mathbb{P}(x \in \Phi, x+h \in \Psi) & \\
& -\mathbb{P}(x \in \Phi) \cdot \mathbb{P}(x+h \in \Psi)
\end{aligned}
$$

for $h \in \mathbb{R}^{2}$.

Let be given three random closed sets $\Phi, \Psi$ and $\Xi$ in $\mathbb{R}^{2}$, where $\Psi$ is independent of $\Xi$. We introduce two further random sets $\Phi^{\prime}$ and $\Psi^{\prime}$ defined as the intersections $\Phi^{\prime}=\Phi \cap \overline{\Xi^{c}}$ and $\Psi^{\prime}=\Psi \cap \Xi$, respectively, where $\overline{\Xi^{c}}$ is the topological closure of the complement of $\Xi$. Obviously, $\Phi^{\prime}$ and $\Psi^{\prime}$ depend on each other even if $\Phi$ and $\Psi$ are independent random sets. In this respect we formulate 'conditionally independent infestation', given that $\Phi$ is independent of $\Psi$.

In the context of bark beetle infestation, the specific interpretation of the random sets is as follows:

$\Phi^{\prime}$ - the area of infestation in the previous year (source, red area in Fig. 1),

$\Psi^{\prime}$ - the area of infestation in the subsequent year (target, blue area),

$\Xi$ - the area of trees which are susceptible for infestation (spruce trees, green and blue areas).

The sets $\Phi$ and $\Psi$ are auxiliary quantities introduced in order to formulate independent random infestation. Even for independent $\Phi$ and $\Psi$, the sets $\Phi^{\prime}$ and $\Psi^{\prime}$ depend on each other. (The infestation is called conditionally independent, given that $\Phi$ is independent of $\Psi$.)

Assume that $\Phi$ and $\Psi$ are not observable, i.e., information on $\Phi$ and $\Psi$ may be available only indirectly from the observation of $\Phi^{\prime}, \Psi^{\prime}$ and $\Xi$. Independence of $\Phi$ and $\Psi$ yields

$$
\begin{aligned}
\mathbb{P}\left(x \in \Phi^{\prime}, y \in \Psi^{\prime}\right) & =\mathbb{P}\left(x \in \Phi^{\prime}, y \in \Xi, y \in \Psi\right) \\
& =\mathbb{P}\left(x \in \Phi^{\prime}, y \in \Xi\right) \mathbb{P}(y \in \Psi)
\end{aligned}
$$

for all $x, y \in \mathbb{R}^{2}$. In terms of normalized crosscovariance functions, the last equation can be rewritten as

$$
\frac{\operatorname{cov}_{\Phi^{\prime}, \Psi^{\prime}}(y-x)}{\mathbb{P}\left(x \in \Phi^{\prime}\right) \mathbb{P}\left(y \in \Psi^{\prime}\right)}=\frac{\operatorname{cov}_{\Phi^{\prime}, \Xi}(y-x)}{\mathbb{P}\left(x \in \Phi^{\prime}\right) \mathbb{P}(y \in \Xi)} .
$$




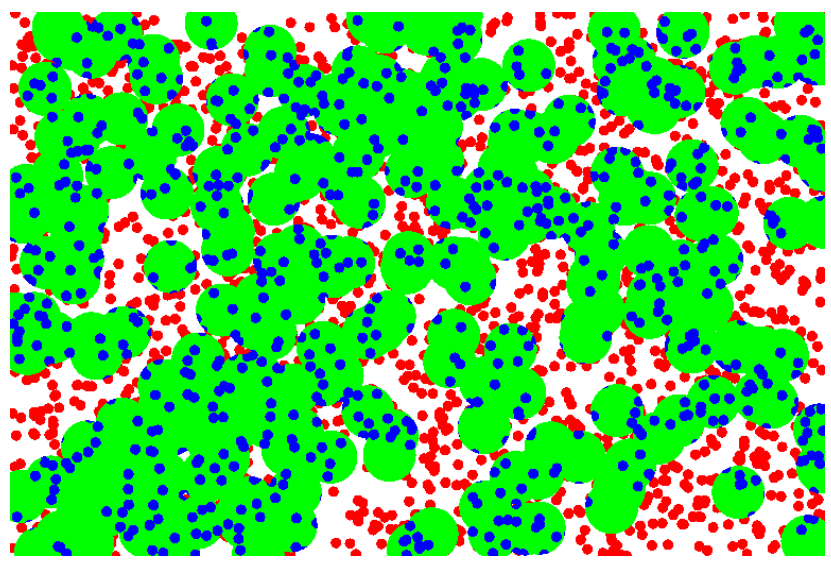

Fig. 2. Simulation of a structure with a complete spatial random infestation. The colors have been chosen as in Fig. 1. In this case $\Phi, \Psi$ and $\Xi$ are independent Boolean models with discs of constant diameter.

In the case of isotropy, both sides of Eq. 2 depend on the radial coordinate $r=\|y-x\|$ only. Let $s_{1}(r)$ be the difference of the left- and the right-hand side of Eq. 2,

$$
s_{1}(r)=\frac{\operatorname{cov}_{\Phi^{\prime}, \Psi^{\prime}}(y-x)}{\mathbb{P}\left(x \in \Phi^{\prime}\right) \mathbb{P}\left(y \in \Psi^{\prime}\right)}-\frac{\operatorname{cov}_{\Phi^{\prime}, \Xi}(y-x)}{\mathbb{P}\left(x \in \Phi^{\prime}\right) \mathbb{P}(y \in \Xi)},
$$

then the relationship $s_{1}(r)=0$ for all $r \geq 0$ is a necessary (but not sufficient) condition for the random sets $\Phi$ and $\Psi$ to be independent of each other. Deviations from 0 indicate dependence (Rack et al., 2008; Ohser and Schladitz, 2009, Section 6.3.3). Estimates of $s_{1}(r)$ characterize the strength of the dependence between the source and the target of infestation depending on the distance $r$.

The interpretation with respect to bark beetle infestation is as follows: Assume that the bark beetle individuals infest subareas of the susceptible forest independent of their source, then $s_{1}(r)$ would vanish for all $r>0$ (complete spatially random infestation) as in Fig. 2. Spatial aggregation between source and target infestation is indicated by $s(r)>0$ for some $r$.

Second-order characteristics (such as crosscorrelations) or their counterparts in frequency space can be measured from images of random structures obtained from various sources (Frank, 1980; Manolakis and Proakis, 1996). The fast Fourier transform (FFT) and sophisticated algorithms for its computation (Marcotte, 1996; Frigo and Johnson, 1998) allow calculating these quantities fast and efficiently. The idea to estimate the auto-covariance function (and other second order characteristics) via the Fourier transform has been around for some time (Debye et al., 1957; Ohser and Mücklich, 2000, chap. 5; Torquato, 2002). A sound mathematical basis for the computation of the auto-covariance function and its counterpart - the so-called Bartlett spectrum - is provided in Koch et al. (2003) and Ohser et al. (2005).

In order to give an estimator of the crosscovariance function we follow the ideas of Rack et al. (2008). Denote by $\mathbb{C}$ the space of complex numbers, and let $f$ be a function $f: \mathbb{R}^{2} \mapsto \mathbb{C}$ belonging to the quotient space $L^{1}\left(\mathbb{R}^{2}\right)=\mathscr{L}^{1}\left(\mathbb{R}^{2}\right) / \mathscr{N}$, where $\mathscr{L}^{1}\left(\mathbb{R}^{2}\right)$ is the set of functions with $\int_{\mathbb{R}^{2}}|f(x)| d x<\infty$ and $\mathscr{N}$ is the set of functions with $f=0$ almost everywhere. Then the Fourier transform $\hat{f}=\mathscr{F} f$ of $f$ is defined by

$$
\mathscr{F} f(\xi)=\frac{1}{2 \pi} \int_{\mathbb{R}^{2}} f(x) \mathrm{e}^{-\mathrm{i} \xi x} \mathrm{~d} x, \quad \xi \in \mathbb{R}^{2} .
$$

Analogously, the inverse Fourier transform $\overline{\mathscr{F}} f$ of $f$ is defined by

$$
\overline{\mathscr{F}} f(x)=\frac{1}{2 \pi} \int_{\mathbb{R}^{2}} f(\xi) \mathrm{e}^{\mathrm{i} x \xi} \mathrm{d} \xi, \quad x \in \mathbb{R}^{2} .
$$

The cross-covariance function is continuous but in general not positive definite. Hence, one can not apply Bochners theorem (Schempp and Dreseler, 1980, Part II, Satz 3.7 and Satz 3.2), in order to introduce a spectral measure associated with the cross-covariance function, and until now it is not known if there exists any counterpart of the cross-covariance function in the frequency space.

Let $\Phi$ and $\Psi$ be macroscopically homogeneous random sets with positive area fractions, and let $W$ be a compact observation window with non-empty interior containing the origin (the complement of the grey frame in Fig. 1). In order to give an estimator of the cross-covariance function of two macroscopically homogeneous random sets $\Phi$ and $\Psi$, we introduce the windowed functions

$$
\varphi_{W}(x)=\mathbf{1}_{W}(x)\left(\mathbf{1}_{\Phi}(x)-A_{A}(\Phi)\right), \quad x \in \mathbb{R}^{2}
$$

and

$$
\psi_{W}(x)=\mathbf{1}_{W}(x)\left(\mathbf{1}_{\Psi}(x)-A_{A}(\Psi)\right), \quad x \in \mathbb{R}^{2}
$$

associated with $\Phi$ and $\Psi$, respectively, where $\mathbf{1}$ is the indicator function. The boundedness of $W$ ensures the integrability of $\varphi_{W}$ and $\psi_{W}$, and hence, their Fourier transforms $\hat{\varphi}_{W}$ and $\hat{\psi}_{W}$ exist almost surely. Furthermore, let $c_{W}=\mathbf{1}_{W} * \mathbf{1}_{\breve{W}}$ denote the window function of $W$, where $*$ is the convolution and $\breve{W}$ is the reflection of $W$ at the origin. Furthermore, let $\overline{\hat{\varphi}}$ denote the complex conjugate of $\hat{\varphi}$. Then, analogously to the estimator of the auto-covariance function of a random set as described in Koch et al. (2003) and Ohser et al. 
(2005), the cross-covariance function of $\Phi$ and $\Psi$ can be estimated via

$$
\operatorname{cov}_{\Phi, \Psi}(x) \approx \frac{2 \pi \overline{\mathscr{F}}\left(\left|\hat{\hat{\varphi}}_{W} \cdot \hat{\psi}_{W}\right|\right)(x)}{c_{W}(x)}
$$

for all $x$ with $c_{W}(x)>0$. Here $\approx$ means that the expression on the right-hand side is an unbiased estimator for $\operatorname{cov}_{\Phi, \Psi}(x)$.

\section{DISTANCE METHOD}

Let $\|x-y\|$ denote the Euclidean distance of two points $x, y \in \mathbb{R}^{2}$. The shortest distance $\operatorname{dist}(x, \Phi)=$ $\inf \{\|x-y\|: y \in \Phi\}$ between a given point $x \in \mathbb{R}^{2}$ and the random set $\Phi$ is a random variable and the mapping dist $(\cdot, \Phi)$ can be considered as a random field. If $\Phi$ is macroscopically homogeneous then so is $\operatorname{dist}(\cdot, \Phi)$, i.e., the distribution of dist $(x, \Phi)$ is independent of the position $x$.

We consider the probability that the distance $\operatorname{dist}(x, \Phi)$ is less than a given value $r \geq 0$ given that $x$ belongs to $\Psi$. Let $A \oplus B=\{x+y: x \in A, y \in B\}$ denote the Minkowski sum of the sets $A, B \subset \mathbb{R}^{2}$. For the particular case of a ball $B_{r}$ with radius $r$ centered at the origin, it holds $A \oplus B_{r}=\left\{x \in \mathbb{R}^{2}: \operatorname{dist}(x, A) \leq r\right)$. If $\mathbb{P}(x \in \Psi)>0$ then

$$
\begin{aligned}
\mathbb{P}(\operatorname{dist}(x, \Phi) & \leq r \mid x \in \Psi) \\
& =\frac{\mathbb{P}(\operatorname{dist}(x, \Phi) \leq r, x \in \Psi)}{\mathbb{P}(x \in \Psi)} \\
& =\frac{\mathbb{P}\left(x \in\left(\Phi \oplus B_{r}\right) \cap \Psi\right)}{\mathbb{P}(x \in \Psi)}
\end{aligned}
$$

for all $x \in \mathbb{R}^{2}$ and $r \geq 0$. In the case of macroscopic homogeneity the conditional probability considered above is independent of the position $x$ and it holds

$$
\begin{aligned}
\mathbb{P}(\operatorname{dist}(x, \Phi) \leq r \mid x \in \Psi) & \\
= & \frac{A_{A}\left(\left\{x \in \mathbb{R}^{2}: \operatorname{dist}(x, \Phi) \leq r\right\} \cap \Psi\right)}{A_{A}(\Psi)} \\
= & \frac{A_{A}\left(\left(\Phi \oplus B_{r}\right) \cap \Psi\right)}{A_{A}(\Psi)}, \quad r \geq 0 .
\end{aligned}
$$

In the following we write $F_{\Phi, \Psi}(r)=\mathbb{P}(\operatorname{dist}(x, \Phi) \leq$ $r \mid x \in \Psi)$.

Again, we derive a relationship which can serve as a condition for independence of random sets. As before we assume that $\Psi$ is independent of $\Phi$ and $\Xi$ with $0<A_{A}(\Xi)<1$ and $A_{A}(\Psi)>0$. As above we define $\Phi^{\prime}=\Phi \cap \overline{\Xi^{c}}$ and $\Psi^{\prime}=\Psi \cap \Xi$, then from the independence of $\Psi$ and $\Xi$ it follows

$$
\begin{aligned}
F_{\Phi^{\prime}, \Psi^{\prime}}(r) & =\mathbb{P}\left(\operatorname{dist}\left(x, \Phi^{\prime}\right) \leq r \mid x \in \Psi^{\prime}\right) \\
& =\frac{\mathbb{P}\left(\operatorname{dist}\left(x, \Phi^{\prime}\right) \leq r, x \in \Psi, x \in \Xi\right)}{\mathbb{P}(x \in \Psi, x \in \Xi)} \\
& =\frac{\mathbb{P}\left(\operatorname{dist}\left(x, \Phi^{\prime}\right) \leq r, x \in \Xi\right)}{\mathbb{P}(x \in \Xi)} \\
& =F_{\Phi^{\prime}, \Xi}(r), \quad r>0 .
\end{aligned}
$$

Define $f_{., .}(r)=\frac{1}{2 \pi} \frac{\mathrm{d}}{\mathrm{d} r} F_{.,}(r)$. Then the difference

$$
s_{2}(r)=f_{\Phi^{\prime}, \Psi^{\prime}}(r)-f_{\Phi^{\prime}, \Xi}(r), \quad r \geq 0,
$$

can be used to check independence of $\Phi$ and $\Psi$. Stochastic dependence of $\Phi$ and $\Psi$ can be assumed if there exist values $r>0$ for which estimates of $s_{2}(r)$ 'significantly' differ from 0.

In principle, the function $F_{\Phi, \Psi}$ can be estimated using either Eq. 3 or Eq. 4. Because of the huge amount of image data, we start from Eq. 3 and apply a method proposed by Rack et al. (2008) and Ohser and Schladitz (2009, Section 5.5.2), where similar to the FFT for cross-correlation, the Euclidean distance transform (EDT) is used as tool allowing a fast computation of quantities like the spherical contact distribution function (Mayer, 2004); we apply Cuisenaire's algorithm which is about linear in time (Cuisenaire, 1999).

Assume that both $\Phi$ and $\Psi$ are observed through the same window. The distance $\operatorname{dist}(\cdot, \Phi)$ is closely related to the $\mathrm{EDT}_{\Phi^{c}}$ of $\Phi^{c}$, which maps to each point of $\mathbb{R}^{2}$ its shortest distance to $\Phi$,

$$
\begin{aligned}
\mathrm{EDT}_{\Phi^{c}}: \mathbb{R}^{2} & \mapsto[0, \infty) \\
x & \mapsto \operatorname{dist}(x, \Phi) .
\end{aligned}
$$

Now the EDT is masked with the random set $\Psi$ as well as with the reduced window $W \ominus B_{r}$, where $\ominus$ denotes the Minkowski subtraction defined by $A \ominus B=$ $\left(A^{c} \oplus B\right)^{c}$ for sets $A, B \subseteq \mathbb{R}^{2}$. This means, we consider the product

$$
\mathrm{EDT}_{\Phi^{c}} \cdot \mathbf{1}_{\Psi} \cdot \mathbf{1}_{W \ominus B_{r}} .
$$

The observation of distances less than $r$ in the reduced window is free of edge effects (minus sampling). Hence, for known area density $A_{A}(\Psi)$ the ratio

$$
\frac{A\left(\left\{x \in \mathbb{R}^{2}:\left(\mathrm{EDT}_{\Phi^{c}} \cdot \mathbf{1}_{\Psi} \cdot \mathbf{1}_{W \ominus B_{r}}\right)(x) \leq r\right\}\right)}{A_{A}(\Psi) \cdot A\left(W \ominus B_{r}\right)}
$$

is an unbiased estimator of $F_{\Phi, \Psi}(r)$ for those $r$ with $A\left(W \ominus B_{r}\right)>0$, i.e., $A\left(W \ominus B_{r}\right)$ is a window function appropriately chosen for the distance $r$. If also $A_{A}(\Psi)$ is estimated from the image data then 
the above estimator is ratio-unbiased, i.e., $F_{\Phi, \Psi}(r)$ is equal to the ratio of the expectation of the denominator and the expectation of the numerator. Note that the distance values obtained from a discrete EDT must be corrected. In the case of square pixels the half pixel size must be subtracted from the obtained distance values (Rack et al., 2008).
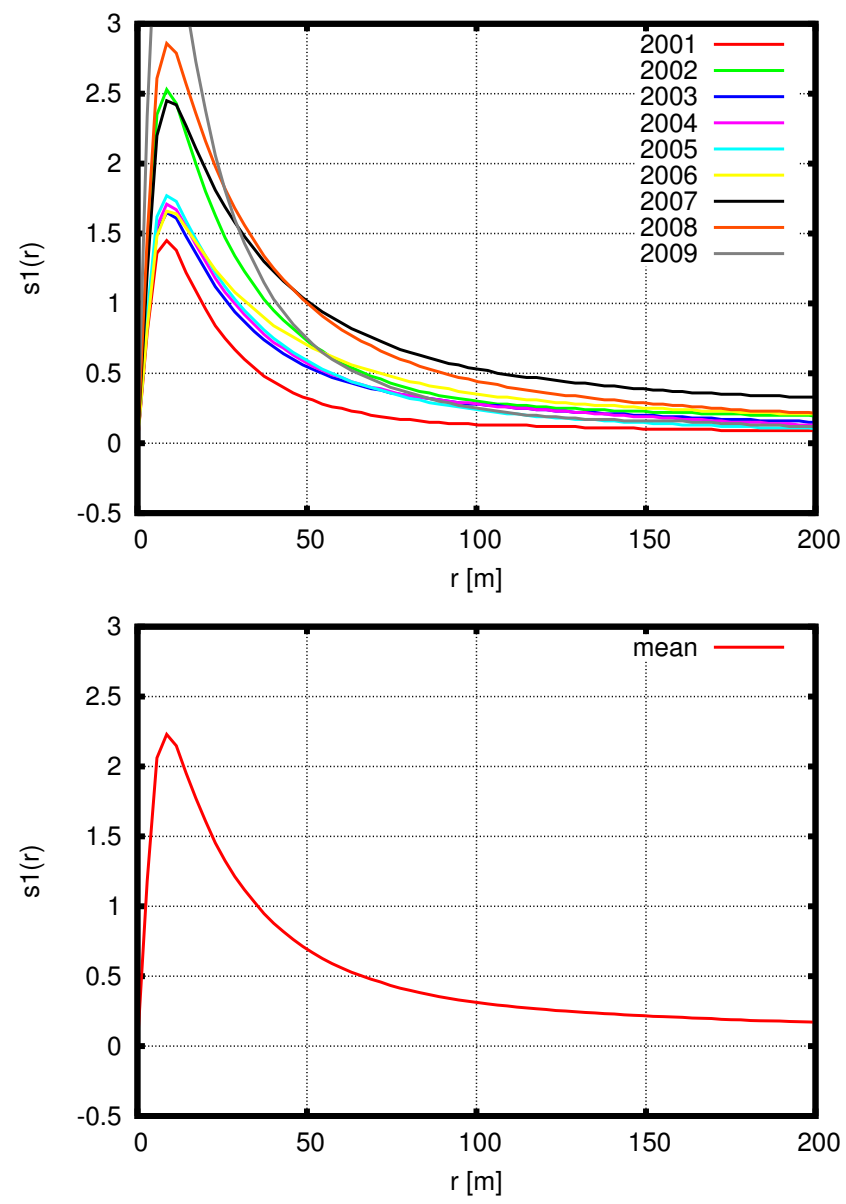

Fig. 3. The function $s_{1}(r)$ for the years 2001 to 2009 (on top indicated by the years of source) and the corresponding mean of $s_{1}(r)$ (bottom).

\section{APPLICATION TO BARK BEETLE INFESTATION DATA}

The data obtained by CIR image interpretation (see Kautz et al., 2011 for details) provide information on the observation window, the area of susceptible trees, and the area of newly emerged infestations for 10 subsequent years (2001-2010). The area of infested trees (not removed from the forest) of the previous year is considered as the source for subsequent infestation. Clearly, those infestation patches where the trees are removed by forest management cannot serve as a source.

Each of the images consists of $6620 \times$ 4803 pixels with an uniform pixel size of $2.87 \mathrm{~m}$. Image processing and analysis are made with MATLAB developed by MathWorks Inc. and the $\mathrm{C}++$ library MAVIlib created at Fraunhofer ITWM, Department of Image Processing (2011).

The results of computation are shown in Figs. 3 and 4. Comparing $s_{1}(r)$ and $s_{2}(r)$, we observe that the shapes of the curves are very similar, although the order of the curves differs. As it has been expected, for large distances $r$ both functions are decreasing and the probability that infestation occurs more distant than $200 \mathrm{~m}$ away from potential source patches is very low. Further, we were interested in how our results depend on the spatial association and the abundance (i.e., intensity) of source patches. The spatial association of source patches was specified by the common Moran's I, an index with values theoretically ranging from -1 (indicating regularity) over 0 (completely random distribution) to 1 (strong clustering), see Moran (1950) and Fortin and Dale (2005). Both functions $s_{1}(r)$ and $s_{2}(r)$ are independent of Moran's I of the sources (Spearman's rank correlation test yields $\left|\rho_{s}\right|<0.4$ and $p$-value $>0.4$ for $s_{1}$ and $s_{2}$ with $r=50 \mathrm{~m}$; Table $1)$. The intensity of infestation affects significantly $s_{1}\left(\left|\rho_{s}\right|=0.85 ; p\right.$-value $\left.=0.004\right)$, while $s_{2}$ is less influenced by the intensity $\left(\left|\rho_{s}\right|=0.6, p\right.$-value $=$ 0.088; Table 1). The most obvious difference between both curves is that for small $r$ the function $s_{1}(r)$ is increasing, with a maximum value around $r=10 \mathrm{~m}$. That is because $s_{1}(r)$ depends on the probability that two points of a given distance $r$ belong to the interior of disjoint patches. In contrast to this, for $s_{2}(r)$ the distances are measured from the boundary of the source patches. The outer skeleton of the source patches (i.e., the ridges of the EDT-image) can be seen as bounds of spreading.

Table 1. Numerical data for evaluation of bark beetle infestation.

\begin{tabular}{ccccc}
\hline year & $\begin{array}{c}\text { source } \\
\text { area [ha] }\end{array}$ & $\begin{array}{c}\text { Moran's } \\
\text { I }\end{array}$ & $s_{1}(50 \mathrm{~m})$ & $s_{2}(50 \mathrm{~m})$ \\
\hline 2001 & 44.81 & 0.76 & 0.32 & 9.41 \\
2002 & 111.11 & 0.74 & 0.64 & 2.63 \\
2003 & 71.95 & 0.47 & 0.55 & 6.14 \\
2004 & 138.35 & 0.57 & 0.58 & 4.22 \\
2005 & 245.74 & 0.58 & 0.59 & 2.49 \\
2006 & 316.14 & 0.65 & 0.70 & 2.15 \\
2007 & 384.61 & 0.59 & 1.03 & 2.86 \\
2008 & 286.95 & 0.62 & 1.02 & 2.59 \\
2009 & 178.03 & 0.76 & 0.75 & 1.88 \\
\hline
\end{tabular}


In the computation of $s_{2}(r)$ it is implicitly assumed that a patch susceptible to infestation can only be infested from the bark beetle subpopulation closest to this patch. In contrast, the computation of $s_{1}(r)$ does not involve any restrictions about the origin of the bark beetles. The individuals spreading out from their source can reach any location of the susceptible forest. Although we obviously do not have knowledge about the true spatial relations between source and target patches, the assumptions implicitely included in the definition of $s_{2}$ are more likely describing the natural dispersal behavior of Ips typographus. However, since the curves of both functions have similar shapes, knowledge on the origin seems not be essential for describing spatial dependence of subsequent infestation patches.

\section{DISCUSSION}

The study demonstrates how image analysis can be applied efficiently on ecological data at various spatial scales. Nevertheless, there are some computational problems that need to be discussed. The algorithmic core of the computation of the function $s_{1}(r)$ from digital images is the FFT and, therefore, the complexity of computing is $\mathscr{O}(n \log n)$, where $n$ is the number of pixels. The FFT supposes periodicity with respect to the window $W$ through which the data are observed, i.e., the mask of spruce trees. However, the forest is not periodic and in particular not $W$ periodic. In higher dimensions the fraction of the data close to the edge of $W$ is considerably larger than in the one-dimensional case. Thus, the kind of windowing as described by Bloomfield (1976) which was successfully applied in one-dimensional cases can lead to a considerable bias in higher dimensions. For the investigations presented in this article we used the edge correction suggested by Koch et al. (2003), which is based on a padding of the image with zeros and choosing an appropriate window function.

The calculation of $s_{2}(r)$ uses a discrete version of the EDT. There are known algorithms for the discrete EDT having a complexity of about $\mathscr{O}(n)$, see Ohser and Schladitz (2009) for an overview. Therefore, computation of $s_{2}(r)$ is faster than $s_{1}(r)-$ which is an important aspect when dealing with a huge data set, as in our case.

Finally, we remark that in the CIR images one can observe a considerable macroscopic inhomogeneity. (In terms of image processing it is called a gradient.) From our experience, the impact of this inhomogeneity on the estimates seems to be negligible. Nevertheless, the characterization of dependence between macroscopically inhomogeneous random sets is an open problem worth being explored.
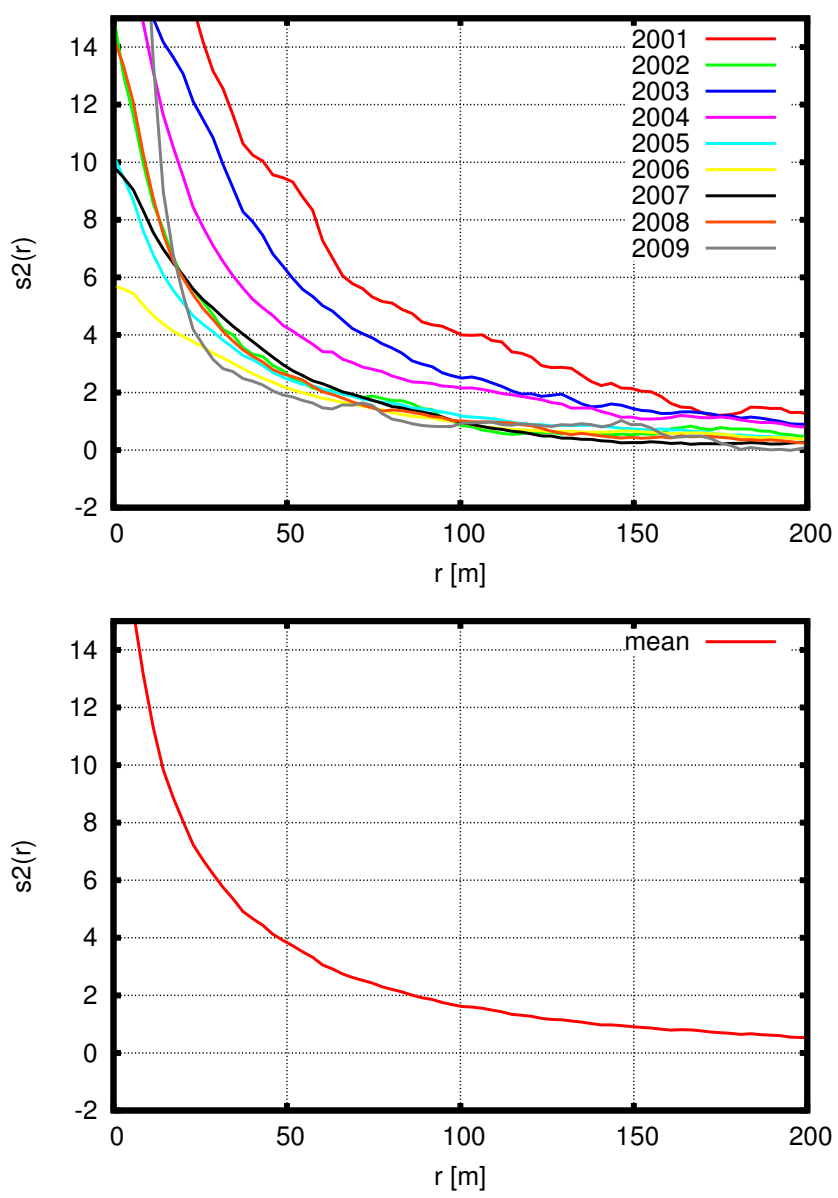

Fig. 4. The function $s_{2}(r)$ for the years 2001 to 2009 (on top the years of source) and the corresponding mean of $s_{2}(r)$ (bottom).

\section{ACKNOWLEDGMENT}

The authors thank Dietrich Stoyan for valueable hints and fruitful discussion, and they are grateful for funding by the Bavarian State Ministry of the Environment and Public Health (UGV-06070204028).

\section{REFERENCES}

Agterberg FP, Fabbri AG (1979). Spatial correlation of stratigraphic units quantified from geological maps. Computers Geosci 4:515-26.

Baddeley AJ, Turner R (2006). Modelling spatial point patterns in R. In: Baddeley AJ, Gregori P, Mateu J, Stoica R, Stoyan D, eds. Case studies in spatial point 
pattern modelling. Lect Notes Stat 185:23-74. New York: Springer.

Bloomfield P (1976). Fourier analysis of time series - An introduction. New York: J. Wiley \& Sons.

Byers JA (2004). Chemical ecology of bark beetles in a complex olfactory landscape. In: Lieutier F, Day KR, Battisti A, Gregoire JC, Evans HF, eds., Bark and wood boring insects in living trees in Europe, a synthesis. Dordrecht: Kluwer Academic Publishers. 89-134.

Cuisenaire O (1999). Distance transformations: fast algorithms and applications to medical image processing. Ph.D. thesis, Université catholique de Louvain, Louvain.

http://www.tele.ucl.ac.be/PEOPLE/OC/these.pdf.

Debye P, Anderson HRJ, Brumberger H (1957). Scattering by inhomogeneous solid. II. The correlation function and its application. J Appl Phys 28:679-83.

Duffy GP, Hughes-Clarke JE (2005). Application of spatial cross correlation to detection of migration of submarine sand dunes: Marine sandware and river dune dynamics. J Geophys Res 110:1-11.

Fortin MJ, Dale MRT (2005). Spatial analysis: a guide for ecologist. Cambridge: Cambridge University Press.

Frank J (1980). The role of correlation techniques in computer image processing. In: Hawkes PW, ed., Computer processing of electron microscope images. Berlin: Springer.

Fraunhofer ITWM, Department of image processing (2011). MAVIlib - modular algorithms for volume images, $\mathrm{C}++$ library. http://www.mavi-3d.de.

Frigo M, Johnson SG (1998). FFTW 2.1.3 (the fastest Fourier transform of the West). http://www.fftw.org/.

Hawkes C (2009). Linking movement behaviour, dispersal and population processes: is individual variation the key? J Anim Ecol 78:894-906.

Heurich M, Ochs T, Andresen T, Schneider T (2010). Object-orientated image analysis for the semi-automatic detection of dead trees following a spruce bark beetle (Ips typographus) outbreak. Eur J Forest Res 129:313-24.

Illian J, Penttinen A, Stoyan H, D. S (2008). Statistical analysis and modelling of spatial point patterns. Statistics in practice. Chichester, New York: J. Wiley \& Sons.

Jakus R (1995). Bark beetle (Col., Scolytidae) communities and host and site factors on tree level in Norway spruce primeval natural forest. J Appl Entomol 119:643-51.

Kautz M, Dworschak K, Gruppe A, Schopf R (2011). Quantifying spatio-temporal dispersion of bark beetle infestations in epidemic and non-epidemic conditions. Forest Ecol Manag 262:598-608.
Koch K, Ohser J, Schladitz K (2003). Spectral theory for random closed sets and estimating the covariance via frequency space. Adv Appl Prob 35:603-13.

Lieutier F, Day KR, Battisti A, Gregoire JC, Evans HF (2004). Bark and wood boring insects in living trees in Europe, a synthesis. Dordrecht: Kluwer Academic Publishers.

Ma J, Zeng D, Chen H (2006). Spatial-temporal crosscorrelation analysis: A new measure and a case study in infectious disease informatics. In: Mehrotra S, Zeng DD, Chen H, Thuraisingham BM, Wang FY, eds. Proc IEEE Int Conf Intell Secur Inform, ISI 2006. May 23-24. San Diego, CA, USA. Lect Notes Comput Sci 3975:542-7. Berlin, Heidelberg: Springer,

Manolakis D, Proakis J (1996). Digital signal processing. Prentice-Hall, 3rd ed.

Marcotte D (1996). Fast variogram computation with FFT. Computers Geosci 22:1175-86.

Mayer J (2004). A time-optimal algorithm for the estimation of contact distribution functions of random sets. Image Anal Stereol 23:177-83.

Moran PAP (1950). Notes on continous stochastic phenomena. Biometrika 37:17-23.

Netherer S, Nopp-Mayr U (2005). Predisposition assessment systems (PAS) as supportive tools in forest management - rating of site and stand-related hazards of bark beetle infestation in the High Tatra Mountains as an example for system application and verification. Forest Ecol Manag 207:99-107.

Ohser J, Mücklich F (2000). Statistical analysis of microstructures in materials science. Chichester, New York: J Wiley \& Sons.

Ohser J, Schladitz K (2009). 3D images of materials structures - processing and analysis. Weinheim, Berlin: Wiley VCH.

Ohser J, Schladitz K, Koch K, Nöthe M (2005). Diffraction by image processing and its application in materials science. Z Metallkunde 96:731-7.

Rack A, Helfen L, Baumbach T, Kirste S, Banhart J, Schladitz K, Ohser J (2008). Analysis of spatial cross-correlations in multi-constituent volume data. J Microscopy 232:282-92.

Reich RM, Czapalewski RL, Bechtold WA (1994). Spatial cross-correlation of undisturbed, natural shortleaf pine stands in northern Georgia. Environ Ecol Stat 1:201-17.

Schempp W, Dreseler B (1980). Einführung in die harmonische Analyse. Stuttgart: Teubner.

Schneider R, Weil W (2008). Stochastic and integral geometry. Probability and its application. Heidelberg: Springer.

Stoyan D (1984a). Correlations of the marks of marked point 
processes - statistical inference and simple models. J Inf Process Cybern 20:285-94.

Stoyan D (1984b). On correlations of marked point processes. Math Nachr 116:197-207.

Stoyan D, Ohser J (1982). Correlations between planar random structures, with an ecological application. Biometrical J 24:631-47.

Stoyan D, Ohser J (1984). Cross-correlation measure of weighted random measures and their estimation. Teor Verojatn Primen 29:338-47.

Torquato S (2002). Random heterogeneous materials: microstructure and macroscopic properties. New York: Springer.
Wallin KF, Raffa KF (2004). Feedback between individual host selection behaviour and population dynamics in an eruptive herbivore. Ecol Monogr 74:101-16.

Wermelinger B (2004). Ecology and management of the spruce bark beetle Ips typographus - a review of recent research. Forest Ecol Manag 202:67-82.

Wichmann L, Ravn HP (2001). The spread of Ips typographus (L.) (Coleoptera, Scolytidae) attacks following heavy windthrow in Denmark, analysed using GIS. Forest Ecol Manag 148:31-9.

Williams WI, Robertson IC (2008). Using automated flight mills to manipulate fat reserves in Douglas-fir beetles (Coleoptera: Curculionidae). Environ Entomol $37: 850-6$.. 